\title{
MicroRNA-449a Inhibits Tumor Metastasis through AKT/ERK $1 / 2$ Inactivation by Targeting Steroid Receptor Coactivator (SRC) in Endometrial Cancer
}

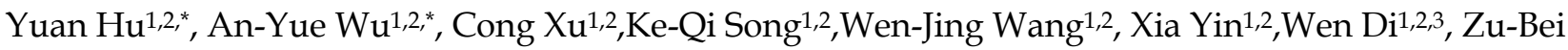 \\ Hong ${ }^{1,2,}$, Li-Hua Qiu ${ }^{1,2,}, \bowtie$ \\ 1. Department of Obstetrics and Gynecology, Ren Ji Hospital, School of Medicine, Shanghai JiaoTong University, Shanghai 200127, China \\ 2. Shanghai Key Laboratory of Gynecologic Oncology, Shanghai 200127, China \\ 3. State Key Laboratory of Oncogenes and Related Genes, Shanghai Cancer Institute, Ren Ji Hospital, School of Medicine, Shanghai Jiao Tong University \\ * Co-first authors: these authors contributed equally to this work. \\ $\triangle$ Corresponding authors: Zu-Bei Hong, Department of Obstetrics and Gynecology, Ren Ji Hospital, 160 Pujian Road, Pu-dong District, Shanghai 200127, China. \\ Phone: +86-21-58752345, Fax: +86-21- 58395057. E-mail: hongzubei@126.com. Li-Hua Qiu, Department of Obstetrics and Gynecology, Ren Ji Hospital, 160 Pujian
} Road, Pu-dong District, Shanghai 200127, China. Phone: +86-21-58752345, Fax: +86-21- 58395057. E-mail: lilyqiulh@126.com

( $)$ Ivyspring International Publisher. This is an open access article distributed under the terms of the Creative Commons Attribution (CC BY-NC) license (https://creativecommons.org/licenses/by-nc/4.0/). See http://ivyspring.com/terms for full terms and conditions.

Received: 2018.06.08; Accepted: 2018.10.28; Published: 2019.01.01

\begin{abstract}
Endometrial cancer represents the leading frequency in gynecological malignancy in developed countries. Even with early detection, metastasis and recurrence remain the main reasons for a high death rate. MicroRNA-449a (miR-449a) has been reported to function as a tumor suppressor, yet the role of miR-449a in endometrial cancer metastasis has not been investigated. The present study identified that miR-449a was downregulated in advanced endometrial cancer. Overexpression of miR-449a decreased the migration and invasion of KLE and AN3CA endometrial cancer cells. Using luciferase reporter assays, we identified that miR-449a directly targeted the steroid receptor coactivator (SRC) by binding to sites in the 3' untranslated regions. Elevated expressions of SRC have been witnessed in advanced endometrial cancer tissues and have promoted tumor metastasis. We also identified that the suppressive effect of miR-449a on metastasis could be mediated by downregulating SRC and that miR-449a could suppress AKT and ERK1/2 pathway activation in endometrial cancer cells. These findings contribute to the current understanding of the function of miR-449a in endometrial cancer.
\end{abstract}

Key words: Endometrial cancer, MicroRNA-449a, SRC, Metastasis

\section{Introduction}

Endometrial cancer, a tumor originating in the endometrium, is the most common gynecological malignancy and the fourth most common malignancy among women in developed countries. According to the American Cancer Society, about 61,380 new cases of endometrial cancer were diagnosed in America in 2017, and 10,920 patients died of this disease [1]. Most women with endometrial cancer are diagnosed at an early stage, generally after having vaginal bleeding. Although the overall prognosis of endometrial cancer is favorable, some women still have aggressive neoplasms, such as deeply invasive tumors, that increase the risk of progression and death [2]. Therefore, there still exists an urgent need to investigate the mechanism of metastasis and progression of endometrial cancer.

MicroRNAs (miRNAs) are a family of small non-coding RNA of approximately 20-22 nucleotides in length that negatively regulate gene expression by targeting $3^{\prime}$ untranslated regions (3'-UTR) of messenger RNA (mRNAs) at a post-transcriptional level, causing either inhibition of translation or degradation of mRNA [3]. Deregulation of miRNA is associated with metastasis and progression of many 
cancers including endometrial cancer [4]. For example, downregulation of miR-181c is highly related to the recurrence of endometrial adenocarcinoma by enhancing $\mathrm{NOTCH} 2$ expression [5]. Overexpression of miR-194 can suppress the epithelial-mesenchymal transition by targeting Sox3 in endometrial cancer, thus inhibiting the process of malignancy [6]. miR-126 can inhibit the migration and invasion of endometrial cancer cells [7].

Reports have shown that miR-449a acts as a tumor suppressor and its expression is downregulated in several cancer tumors-such as lung cancer [8], gastric cancer [9], and prostate cancer [10]. Its suppressive effects on invasion and/or metastasis of cancers have also been identified. For example, miR-449a inhibits the invasion of lung cancer cells by targeting c-Met [11]. Downregulation of miR-449a promotes prostate cancer metastasis through initiating overexpression of PrLZ [10]. However, there is little research investigating the potential role of miR-449a in endometrial cancer, especially its role in metastasis. In our study, we have focused on the potential impact of miR-449a on migration and invasion of endometrial cancer and have investigated the mechanisms that underline it.

\section{Materials and Methods}

\section{Patients and ethics}

A total of 40 freshly-frozen human endometrial cancer tissue samples were obtained from patients who had undergone tumor debulking at Renji Hospital, School of Medicine, Shanghai Jiao Tong University. The study was approved by the institutional review board of Renji Hospital, School of Medicine, Shanghai Jiao Tong University. Written informed consent was obtained from all patients.

\section{Cell culture}

The human endometrial adenocarcinoma cancer cell lines KLE, AN3CA, HEC-1A and Ishikawa were obtained from the Cell Bank of the Chinese Academy of Sciences (Shanghai, China). Cells were cultured in DMEM/F12 medium (Hyclone) supplemented with $10 \%$ fetal bovine serum (Hyclone) and penicillin/streptomycin (1:100, Sigma) in a humid-atmosphere incubator with $5 \% \mathrm{CO}_{2}$ at $37^{\circ} \mathrm{C}$.

\section{MicroRNA and small interfering RNA (siRNA) transfection}

Between 40 and $50 \%$ of confluent cells were transfected with human miR-449a mimics, inhibitors, or negative controls (NCs), separately. The sequence of mimics, inhibitors and NCs are as follows: mimics: 5'-UGGCAGUGUAUUGUUAGCUGGU-3'; mimics NC: 5'-UUCUCCGAACGUGUCACGUUU-3' inhibi- tors: 5'-ACCAGCUAACAAUACACUGCCA-3', inhibitors NC: 5'-CAGUACUUUUGUGUAGUACAA-3' (IBS company). Between 30 and $40 \%$ of confluent cells were transfected with steroid receptor coactivator (SRC) siRNA or siRNA-NC by Lipofectamine 2000 (Invitrogen) according to the manufacturer's protocol. Total RNA was extracted 24, 48, or 72 hours after transfection, and total cell proteins were extracted 48 hours after transfection.

\section{Quantitative real-time PCR}

Total RNA was isolated using Trizol Reagent (Invitrogen). Mature miR-449a was reverse transcribed with specific reverse transcription primers, quantified with a TaqMan probe, and normalized by U6 small nuclear RNA using TaqMan miRNA assays (Applied Biosystems).

\section{Western blotting}

Whole cell extracts were prepared as described previously, and equal amounts of protein were separated using 8 or $10 \%$ SDS-PAGE and electrotransferred to a polyvinylidene fluoride (PVDF) membrane (Millipore). The membranes were then blocked for one hour at room temperature with a Li-Cor blocking agent (Li-Cor). With constant shaking, the membranes were then incubated with primary antibodies overnight at $4^{\circ} \mathrm{C}$ followed by incubation for hour with the appropriate secondary antibodies labeled with 800IRdye. Immunoreactivity was detected and quantified with the infrared Odyssey imaging System (Li-Cor). SRC(36D10) Rabbit monoclonal antibody (mAb), Phospho-p44/42 mitogen-activated protein kinase (MAPK) (Thr202/Tyr204) Rabbit mAb (\#4370), Phospho-Akt (Ser473/Thr308) Rabbit mAb (\#4060/\#2965), and Akt (pan) Rabbit mAb (\#4691) were purchased from Cell Signaling Technology. P44/42 MAPK Rabbit mAb was obtained from Santa Cruz (sc-33746). GAPDH mouse mAb was purchased from ABmart (\#M20006).

\section{Wound healing assay}

For the wound healing assay, $5 \times 10^{6}$ cells were plated and grown until a confluent state was reached, and cells were then scratched using sterile tips. Cellular migration (toward the scratched area) was evaluated after $24 \mathrm{~h}$ and $48 \mathrm{~h}$.

\section{Migration and invasion assays}

In the migration assay, $4 \times 10^{4}$ cells were harvested and resuspended in serum-free DMEM/F12 medium and were placed into an $8 \mathrm{~mm}$ pore-size polycarbonate membrane Transwell insert (Corning Costar, MD, USA). In the invasion assay, $8 \times 10^{4}$ cells were placed into the upper chambers coated with $50 \mu \mathrm{l}$ of Matrigel (1:5 dilution in a 
serum-free medium). Medium containing $10 \%$ fetal bovine serum was added into the lower chamber. After $24 \mathrm{~h}$ of incubation, cells remaining on the upper surface of the membranes were removed; cells that had invaded through the pore membrane were fixed, stained, and counted under a microscope at 200x magnification. The results were averaged among three independent experiments.

\section{Luciferase reporter assay.}

Cells were transfected into 6-well plates with SRC 3'-UTR luciferase reporter plasmid, or its mutant, using Lipofectamine 2000 (Invitrogen). After $48 \mathrm{~h}$ of co-transfection, Luciferase assays were performed using the Dual-Luciferase ${ }^{\circledR}$ Reporter kit (Promega) according to the manufacturer's instructions. Luciferase activity was standardized relative to renilla activity as a control.

\section{Immunohistochemistry (IHC) and Chromogenic in situ hybridization (CISH)}

IHC was performed using a horseradish peroxidase (HRP) polymer anti-mouse IHC diaminobenzidine (DAB)-based kit (MaxVision, Fuzhou, China) according to the manufacturer's protocol. Antigen retrieval was performed using a borate buffer $(\mathrm{pH}=8)$, followed by incubation in hydrogen peroxide and additional blocking steps. Antibodies were used at 1:50. The IHC was examined and imaged using an OLYMPUS BX51 microscope (Tokyo, Japan) at 1:100 or 1:200. Positive signals were identified by the intense brown labeling of the cell membranes.

$\mathrm{CISH}$ was performed using the hsa-miR-449a probe from Exiqon (mercury LNA detection probe $5^{\prime}$ and 3'-digoxigenin-labeled). The probe was detected using digoxigenin antibody (Abcam), LSAB2 System-HRP (Dako Denmark A/S, Glostrup, Denmark) and liquid DAB+ Substrate Chromogen System (Dako) according to the manufacturer's instruction. The CISH was examined and imaged using an OLYMPUS BX51 microscope (Tokyo, Japan) at $1: 100$ or 1:200. Positive hybridization signals were identified by the intense brown labeling of the cell cytoplasm.

The results of IHC and CISH were independently scored by two pathologists in a blind manner. The scoring was based on the intensity and extent of staining and was evaluated according to the following histological scoring method: 0 , negative staining; 1 , weak staining; 2 , moderate staining; 3 , strong staining. The mean proportion of staining cells per specimen was determined semi-quantitatively and scored as follows: 0 for staining $<1 \%, 1$ for $1-25 \%$, 2 for $26-50 \%, 3$ for $51-75 \%$, and 4 for $>75 \%$ of the examined cells. The histological score (H-score) for each specimen was computed with the formula: $\mathrm{H}$-score $=$ proportion score $\times$ intensity score. A total score between 0 and 12 was calculated and graded as negative $(-$, score: 0$)$, weak $(+$, score: $1-4)$, moderate $(++$, score: $5-8)$ or strong $(+++$, score: $9-12)$.

\section{Statistical analysis}

Statistical analysis was performed with the SPSS 24.0 statistical analysis software package. In each in-vitro experiment, a minimum of three wells/dishes was used and similar results were obtained. Each experiment was repeated a minimum of three times, the mean value of the repetitions was calculated, and this value was used in the statistical analysis. The differences between groups were evaluated using Student's t-test when comparing only two groups, or they were analyzed using one-way ANOVA with post hoc tests when more than two groups were compared. The results of the IHC and CISH were analyzed using the chi-squared or Fisher's exact test. Differences were considered statistically significant at $P<0.05$.

\section{Results}

\section{miR-449a was downregulated in advanced endometrial cancer tissues}

To investigate the role of miR-449a in endometrial cancer with deep insight, we first measured the expression levels of miR-449a in both stage I-II and stage III-IV endometrial tumor tissues. The results showed that the expression of miR-449a was generally lower in stage III-IV tissues compared with the stage I-II samples (Fig. 1A-B, $P<0.05$ ). There were no significant differences in patient age or histological differentiation between the two groups (Fig. 1C). Taken together, our results suggest that downregulation of miR-449a is correlated with increased metastasis of endometrial cancer.

\section{miR-449a suppresses the migration and invasion of endometrial cancer}

We then evaluated the potential effects of miR-449a on migration and invasion in endometrial cancer cells. According to the expression of miR-449a in four endometrial cancer cell lines, we selected KLE and AN3CA cells, which have a relatively low and high expression of miR-449a, respectively (Fig. 2A). To evaluate the potential effects of miR-449a on migration and invasion in endometrial cancer, we transfected KLE cells with miR-449a mimics, and a subsequent qRT-PCR showed a significant increase in expression of miR-449a. However, AN3CA cells transfected with miR-449a inhibitors performed oppositely (Fig. 2B, $P<0.05$ ). 


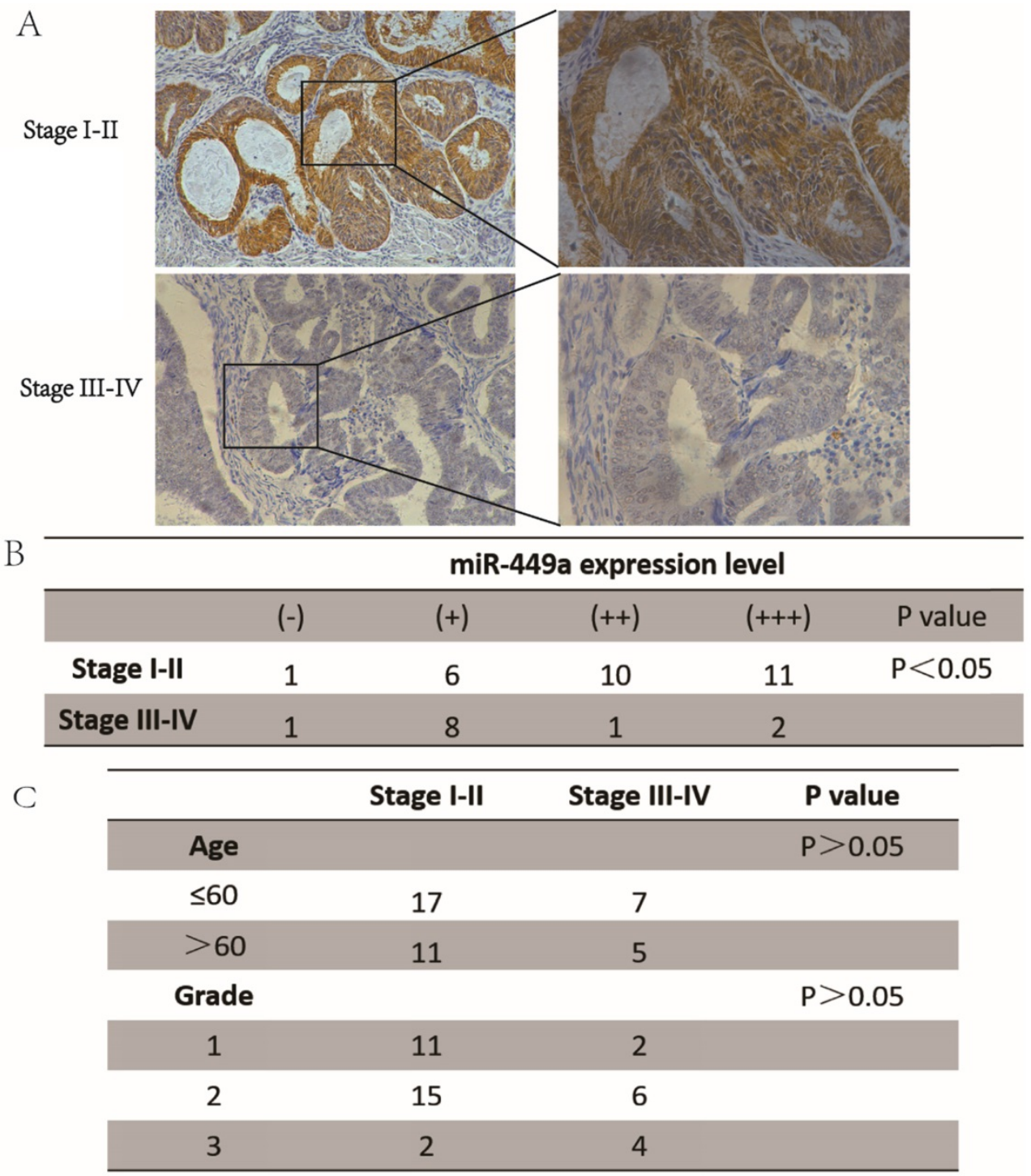

Figure 1. miR-449a was downregulated in advanced endometrial cancer tissues. (A) and (B) Expression of miR-449a in both stages I-II and stages III-IV endometrial tumor tissues by $\mathrm{CISH}$. $\mathrm{H}$-score $=$ proportion score $\times$ intensity score. A total score of between 0 and 12 was calculated and graded as negative $(-$, score: 0$)$, weak $(+$, score: $1-4)$, moderate $(++$, score: $5-8)$ or strong $(+++$, score: $9-12)$. (C) Clinicopathological features of endometrial cancer tissues regarding the relative expression of miR-449a.

Wound healing and Transwell assays were performed to assess the effects of miR-449a on migration and invasion of endometrial cancer cells. The wound healing assay showed that KLE cells transfected with miR-449a mimics moved more slowly compared to the control group (Fig. 2C, $P<0.05)$. However, AN3CA cells transfected with miR-449a inhibitors moved more quickly (Fig. 2D, $P<0.05)$. The wound healing assays imply that miR-449a could suppress the migration of endometrial cancer. In Transwell assays, the exogenous increase of miR-449a expression with mimics decreased the number of migrative and invasive cells significantly in KLE cells (Fig. 2E, $P<0.05)$. However, the decrease of miR-449a expression with inhibitors enhanced the migratory and invasive cells of AN3CA (Fig. 2F, $P<0.05$ ).
SRC is negatively regulated by miR-449a by targeting putative binding sites in the 3'-UTR

To determine the possible target genes of miR-449a in endometrial cancer cells, we used four algorithms-miRanda, Targetscan, miRDB and miRWalk - to analyze potential targets of miR-449a. In the potential target list, SRC was a notably attractive candidate because of its important role in cancer progression. To confirm that miR-449a directly targets SRC, we conducted luciferase reporter assays in which SRC-3'-UTR-wild or SRC-3'-UTR-mut were constructed and inserted into the region downstream of the reporter gene (Fig. 3A). Luciferase reporter assays showed that miR-449a transfection caused a remarkable decrease in luciferase activity containing the wild-type 3'-UTR fragment binding sites (Fig. 3B), thus identifying that SRC is a predicted target of 
miR-449a. It is well known that miRNAs suppress the expression of target genes through translational repression or degradation of a target transcript. Taking this result into account, we further performed western blot assays to assess whether miR-449a has a functional role in the downregulation of endogenous expression of SRC. As shown in Fig. 3C, compared with the control group, the SRC protein was downregulated in the cells transfected with miR-449a mimics, and upregulated in the cells transfected with miR-449a inhibitors. These two assays thus confirm that SRC is a target of miR-449a by bending sites in the 3'-UTR.

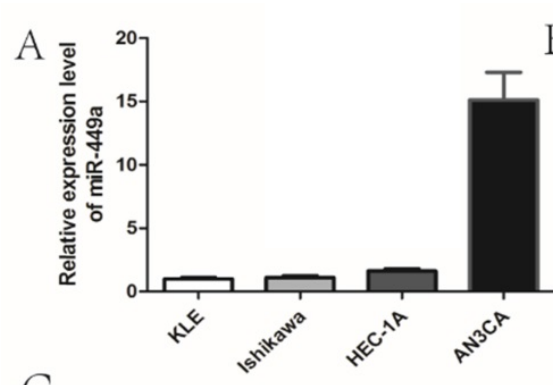

C

$\mathrm{Oh}$
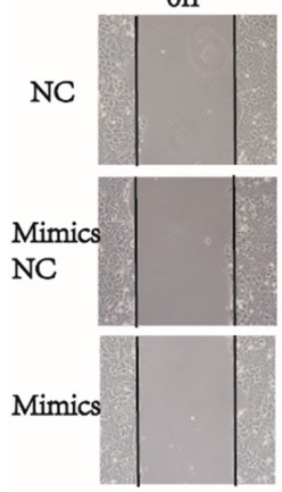

E
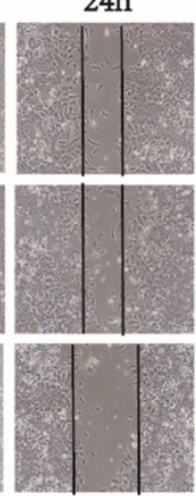

NC
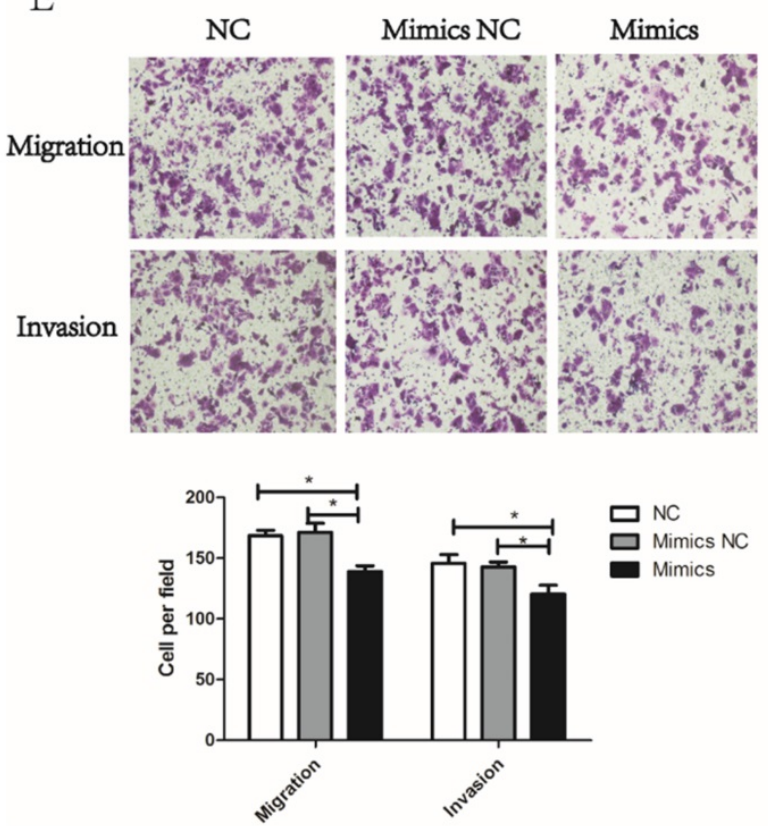

$\mathrm{B}$ 弯

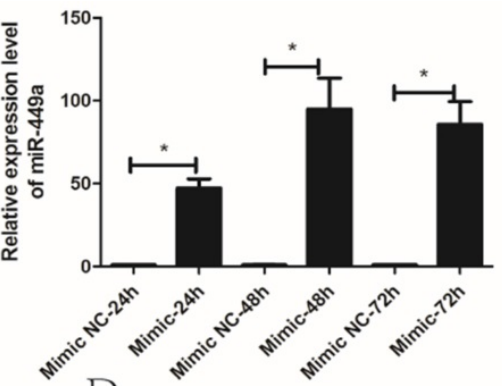

D
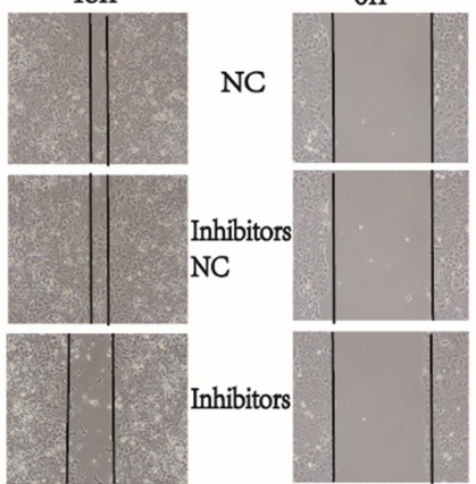

F

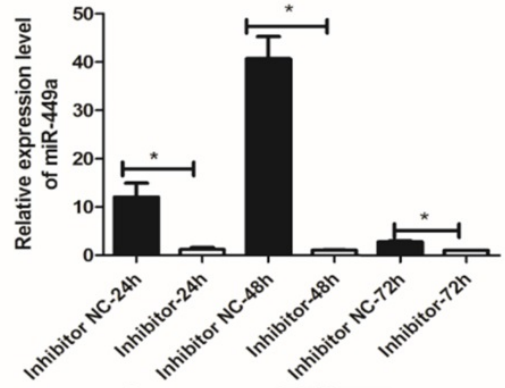

48h
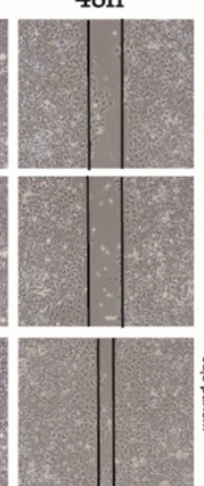

口 ${ }^{\text {m }}$ mimics NC

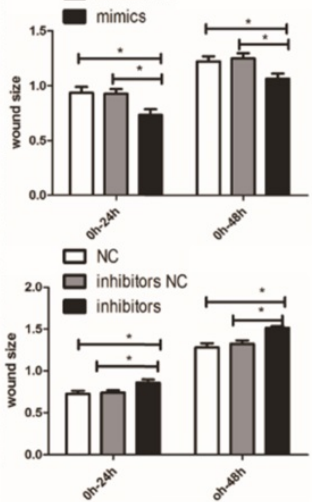

Inhibitors
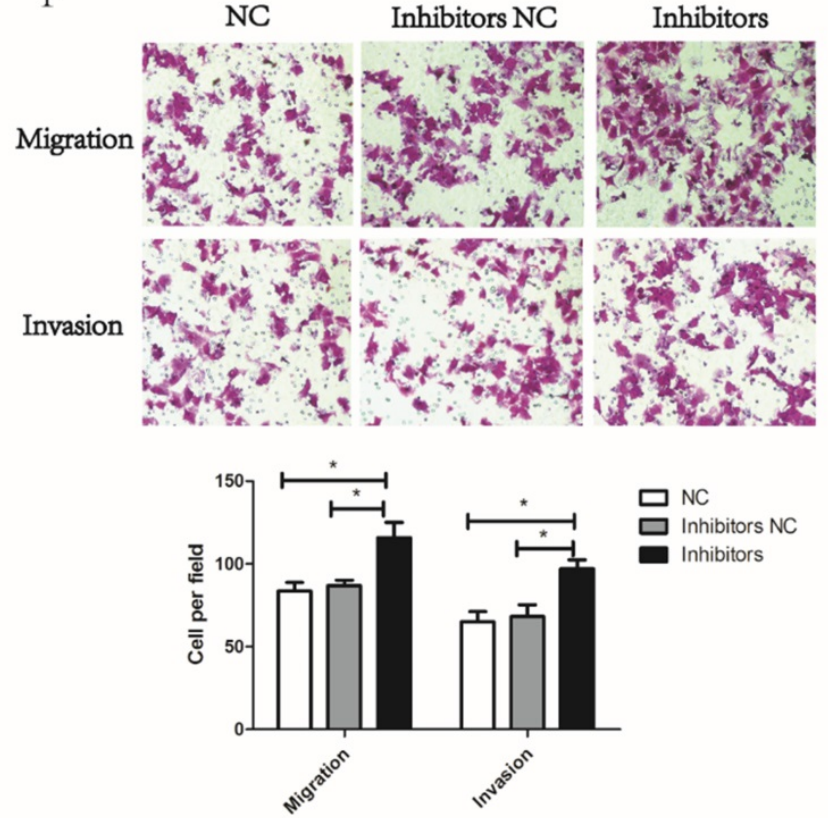

Figure 2. miR-449a suppresses the migration and invasion of endometrial cancer. (A) The expression level of miR-449a in four endometrial tumor cell lines. (B) The expression level of miR-449a in KLE cells transfected with mimics or negative controls (NCs), AN3CA cells with inhibitors or NC was analyzed by qRT-PCR. ${ }^{*} P<0.05$. (C) Wound healing assay of KLE cells transfected with miR-449a mimics or NCs. (D) Wound healing assay of AN3CA cells transfected with miR-449a inhibitors or NCs. ${ }^{*} P<0.05$. (E) Transwell assay of KLE cells transfected with miR-449a mimics or NCs. (F) Transwell assay of AN3CA cells transfected with miR-449a inhibitors or NCs. *P < 0.05. 
A

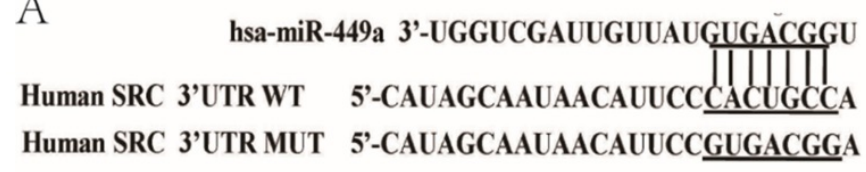

C
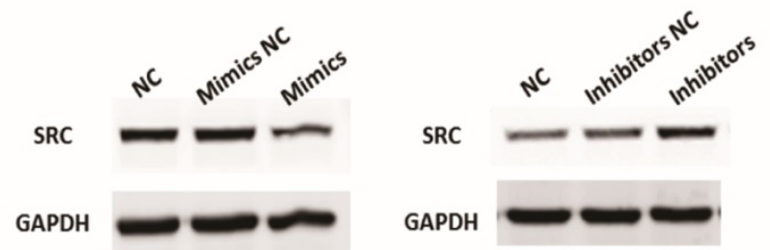

$\mathrm{B}$

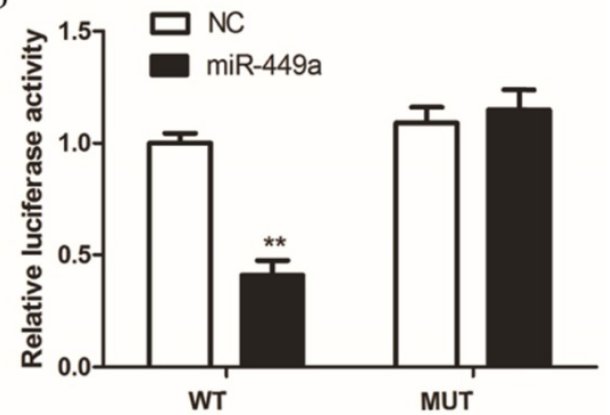

Figure 3. miR-449a downregulate SRC by targeting putative binding sites in the 3'-UTR. (A) Putative miR-449a binding sequence in the 3'-UTR of SRC mRNA (B) Luciferase assay on KLE cells. KLE cells were co-transfected with miR-449a mimics and a luciferase reporter containing the full length of SRC 3'-UTR (WT) or a mutant (Mut) in which the miR-449a-binding site were mutated. Luciferase activities were measured $48 \mathrm{~h}$ hours post transfection. ${ }^{*} P<0.05$. (C) The expression of SRC protein was analyzed by Western blotting in KLE and AN3CA cells transfected with miR-449a mimics or inhibitors.

\section{SRC was upregulated in advanced endometrial cancer tissues and affected migration and invasion in vitro}

Next, we investigated the expression of SRC in endometrial tumor tissues. The results revealed that SRC expression was higher in stage III-IV endometrial tumor tissues compared to stage I-II samples (Fig. 4A-B, $P<0.05$ ), implying that SRC may be correlated with metastasis in endometrial cancer. To further identify the effect of SRC, AN3CA cells were transfected with SRC siRNA. A Western blot assay, shown in Fig. 4C, showed a downregulated expression of SRC when transfected with SRC siRNA. Compared with the control group, the SRC siRNA transfection group experienced a significantly diminished number of migratory and invasive cells through the matrigel (Fig. 4D, $P<0.05$ ). In conclusion, SRC was upregulated in advanced stage endometrial cancer tissues and promoted metastasis in vitro.

\section{Suppressive effect of miR-449a on metastasis of endometrial cancer cells is mediated by downregulating SRC}

To verify whether the metastasis phenotype of endometrial cancer cells could be modulated by SRC, SRC siRNA and SRC plasmids were used to silence and enhance SRC expression, respectively. As shown in Fig. 5A, cotransfection of miR-449a mimics and SRC-plasmids reversed the effects of miR-449a's downregulation on SRC expression. Meanwhile, this cotransfection abolished the suppressive effects of miR-449a on migration and invasion in KLE cells (Fig. 5B, $P<0.05)$. In AN3CA cells, however, cotransfection of miR-449a inhibitors and SRC-siRNA reverted the effects of upregulation of SRC expressions (Fig. 5C) and switched the stimulating effects of miR-449a on migration and invasion (Fig. 5D, $P<0.05$ ). We conclude that SRC is the main mediator through the suppressive effect of miR-449a on metastasis of endometrial cancer cells.
miR-449a suppresses AKT and ERK1/2 pathway activation in endometrial cancer cells

A previous study has shown that SRC activates a cascade of downstream signal pathways including AKT and ERK1/2 pathways. Considering the targeting effect of miR-449a on SRC, we investigated whether miR-449a could affect the above pathways in endometrial cancer. We transfected KLE and AN3CA cells with miR-449a mimics and inhibitors, respectively, and then examined the expression of phosphorylated AKT and ERK1/2 by Western blotting. As shown in Fig. 6, transfection with miR-449a mimics suppressed the expression of phosphorylated AKT and ERK1/2 (Fig. 6A). On the contrary, AN3CA cells, transfected with miR-449a inhibitors, enhanced the expression of phosphorylated AKT and ERK1/2 (Fig. 6B). In conclusion, miR-449a can suppress the activation of AKT and ERK1/2 pathways in endometrial cancer cells.

\section{Discussion}

Endometrial cancer is a common gynecological malignancy among women in Western countries. Even if detected early, the death rate of endometrial cancer has still risen by $2 \%$ per year in the last five years [1], and metastasis and recurrence may be for the main reason behind this increasing death rate. Therefore, a thorough investigation into the mechanisms of endometrial tumor invasion and metastasis may help improve the overall survival of cancer patients. Recent years have witnessed a growing interest in research on miR-449a because of its potential suppressive effects on cancer initiation and progression $[8,10]$. Yet, there is little research on the effects of miR-449a in endometrial cancer. The present study has demonstrated, for the first time, that miR-449a was downregulated in advanced endometrial cancer tissues and could restrain the 


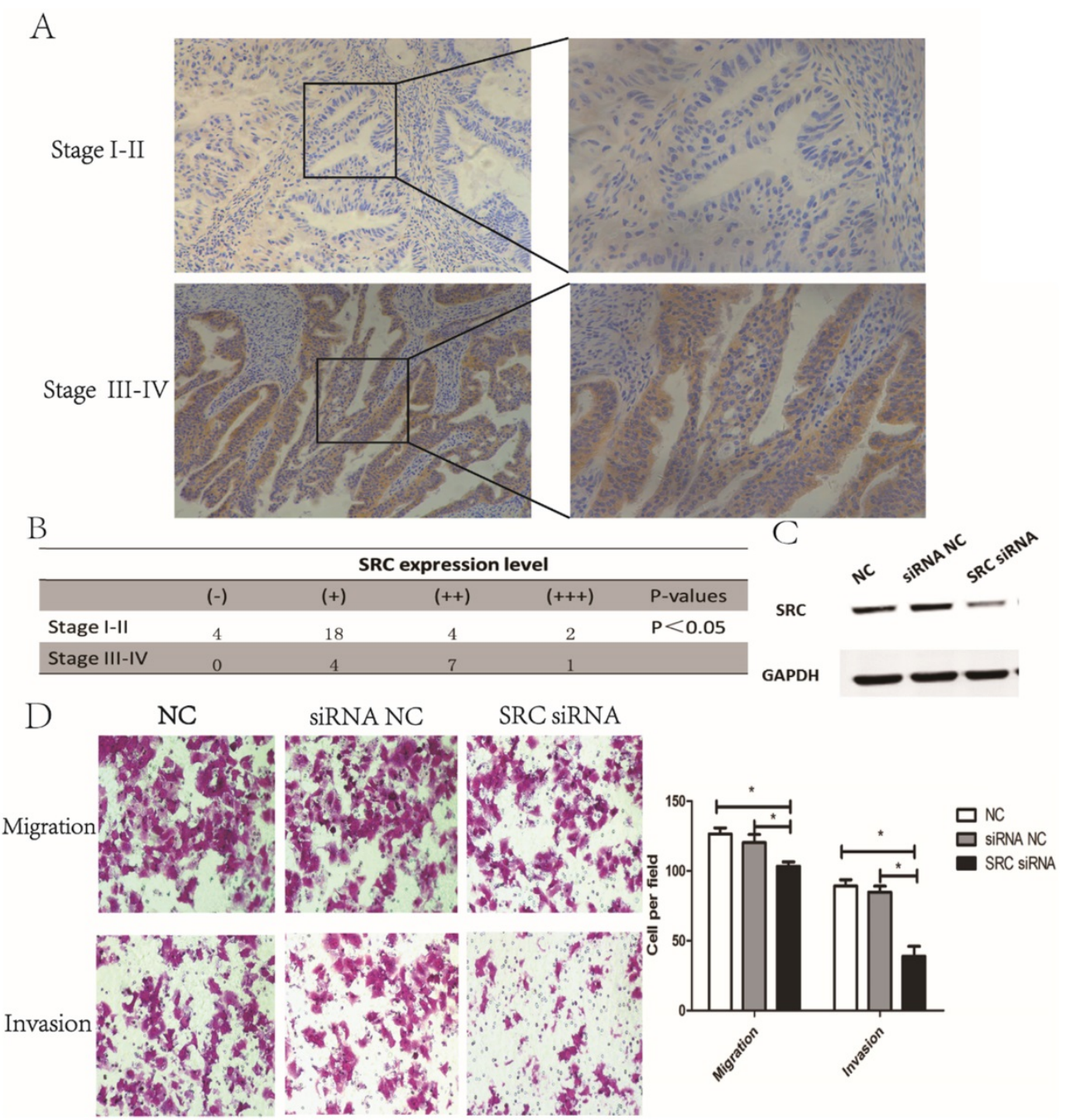

Figure 4. SRC was upregulated in advanced endometrial cancer tissues and affected migration and invasion in vitro. (A) and (B) Expression of SRC in both stage $\mathrm{I}-\mathrm{II}$ and stage III-IV endometrial tumor tissues by IHC. $\mathrm{H}$-score = proportion score $\times$ intensity score. A total score between 0 and 12 was calculated and graded as negative (-, score: 0 ), weak (+, score: $1-4)$, moderate (++, score: $5-8)$ or strong (+++, score: 9-12). (C) The expression of SRC protein was analyzed by Western blotting in AN3CA cells transfected with SRC siRNA. (D) Transwell assay of AN3CA cells transfected with SRC siRNA. * $P<0.05$

migration and invasion of endometrial cancer cells, thus identifying the suppressive role of miR-449a in endometrial cancer (Fig. 1 and Fig. 2).

It is well known that miRNA regulates the expression of genes by binding to the $3^{\prime}$-UTRs of target mRNAs. Reports have shown the potential targets of miR-449a, such as Flot2 [9], Bcl-2 [12], and c-Met [11]. In this study, we used luciferase reporter assays to identify that SRC is a target of miR-449a (Fig. 3 ), and we also demonstrate, using reversion assays, that miR-449a can suppress the migration and invasion of endometrial cancer cells by targeting SRC (Fig. 5).

Coactivators are characterized as a large class of proteins that partner with nuclear receptors (NRs) and other transcription factors to regulate various gene expressions. Without the intermediate coactivators, NRs cannot drive transcription by themselves [13]. SRC, the first identified coactivator, can stimulate the transcriptional activities of the progesterone receptor, estrogen receptor, and other nuclear receptors [14]. Acting in the central position in many signaling pathways, SRC activates a cascade of downstream signal pathways, such as phosphatidylinositide 3-kinase (PI3K)/AKT and MAPK/ERK pathways, and controls multiple cellular processes, especially migration. Because of its powerful regulation and strong connection with NRs, SRC has been recognized as a key oncoprotein in multiple cancers. SRC has been found to be overexpressed in a wide array of cancer types, including breast cancer [15] and prostate cancer [16]. In endometrial cancer, SRC has been found to be overexpressed [17], and this overexpression of SRC has been associated with clinical stage, depth of tumor invasion, and poor survival $[18,19]$. In our study, we have identified that SRC is upregulated in advanced endometrial cancer tissues and contributes to the migration and invasion of tumors cells (Fig. 4). 
A

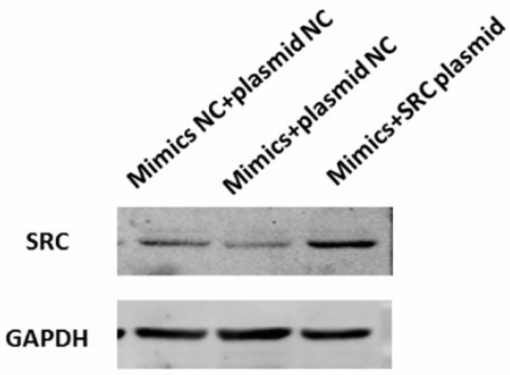

C

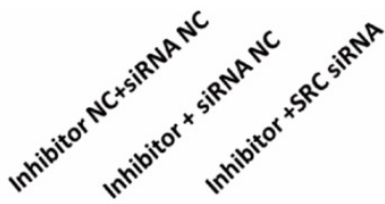

SRC

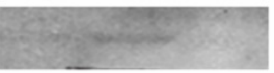

GAPDH

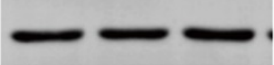

B

Mimics NC+

Plasmid NC

Migration
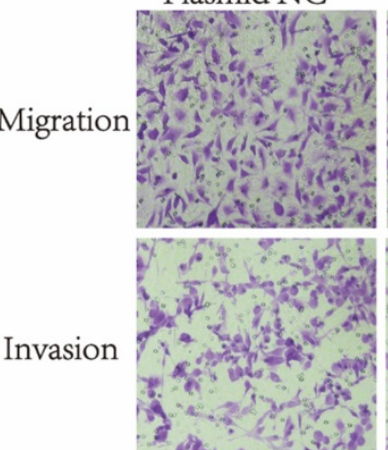

D

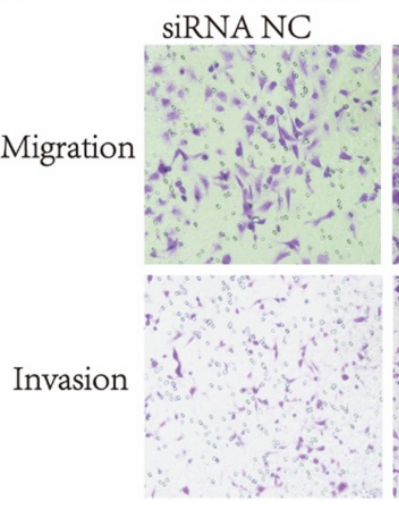

Mimics+

Plasmid NC

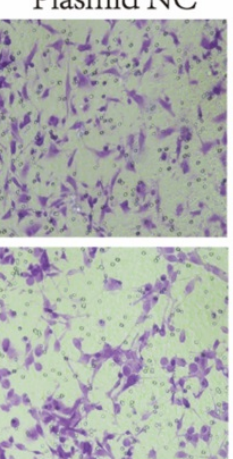

Inhibitors+ siRNA NC

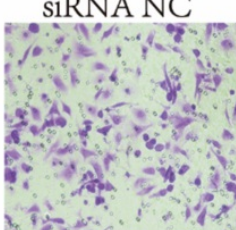

Mimics+
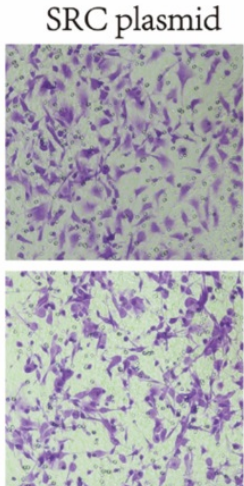

Inhibitors+

SRC siRNA
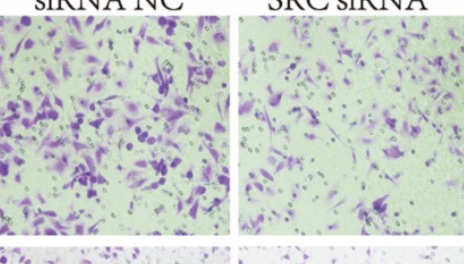
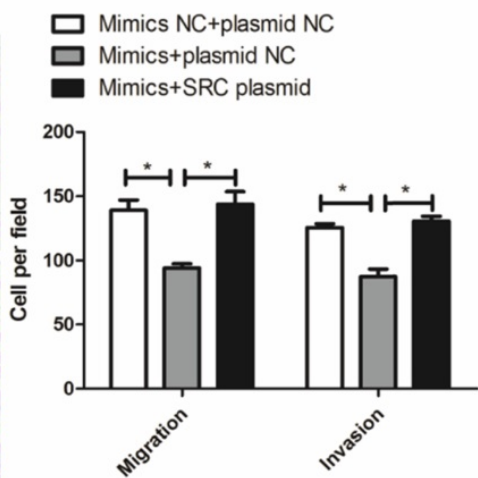

\footnotetext{
$\square$ inhibitors NC+siRNA NC

$\square$ inhibitors+siRNA NC

inhibitors+SRC siRNA
}

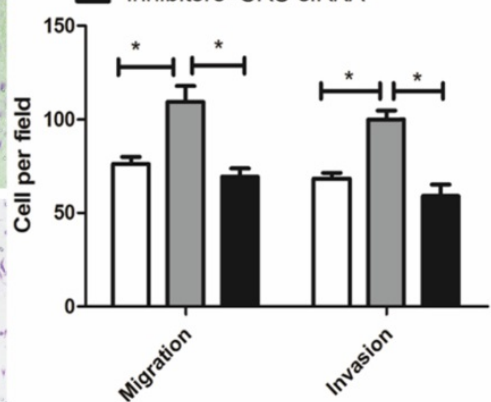

Figure 5. Suppressive effect of miR-449a on metastasis of endometrial cancer cells is mediated by downregulating SRC. (A) The expression of SRC protein was analyzed by Western blotting in KLE cells co-transfected with miR-449a mimics and SRC plasmid. (B) Transwell assay of KLE cells co-transfected with miR-449a mimics and SRC plasmid. ${ }^{*} P<0.05$ (C) The expression of SRC protein was analyzed by Western blotting in AN3CA cells co-transfected with miR-449a inhibitors and SRC siRNA. (D) Transwell assay of AN3CA cells co-transfected with miR-449a inhibitors and SRC siRNA. * $P<0.05$

A

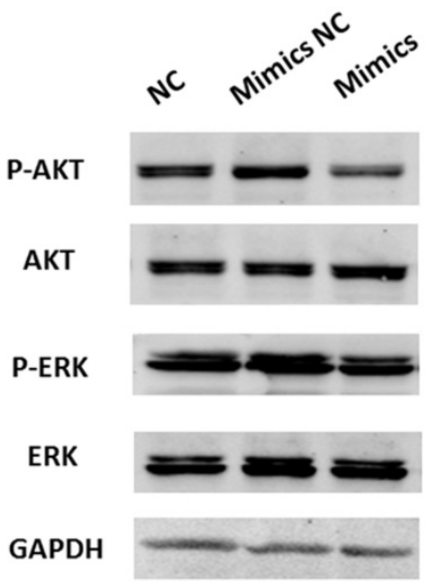

B

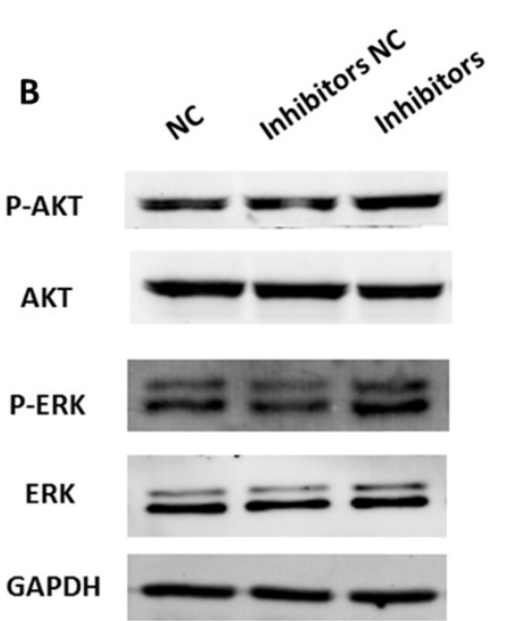

Figure 6. miR-449a suppresses activation of AKT and ERK1/2 pathways in endometrial cancer cells. (A) KLE cells were transfected with miR-449a mimics or negative controls (NCs), AKT and ERK1/2 phosphorylation were analyzed by Western blotting. (B) AN3CA cells were transfected with miR-449a inhibitors or NCs, AKT and ERK $1 / 2$ phosphorylation were analyzed by Western blotting. 
Due to its central position in multiple signal transduction pathways and powerful regulation on tumor progression, SRC has been recognized as a potential target. Many compounds have been developed as inhibitors, such as Gossypol, Bufalin and SI-2 [20]. Gossypol was identified as a potential inhibitor by interfering with the binding between SRC and the estrogen receptor ligand binding domain [21], while Bufalin and SI-2 modulate the transcriptional activity of SRC $[22,23]$. Some phase I and phase II clinical trials are underway, such as trials for Dasatinib, Bosutinib and AZD0530 [24]. However, to date, there are few studies aimed at inhibiting SRC at a post-transcriptional level. In our study, we have first identified that miR-449a could post-transcriptionally decrease the expression of SRC by binding to its 3'-UTR, and this represents a new way of inhibiting SRC.

In conclusion, the present study provides evidence that miR-449a inhibited the migration and invasion of endometrial cancer and its expression was identified inversely correlated with tumor progression. SRC is a potential target of miR-449a by binding to sites in the SRC 3'-UTR and contributes to the metastasis of endometrial cancer. miR-449a could suppress the activation of the AKT and ERK1/2 pathways in endometrial cancer cells. We preliminarily explored miR-449a as a promising therapeutic target and potential inhibitor of SRC.

\section{Acknowledgements}

This work was supported by National Natural Science Foundation of China (81272884), Project of Pudong New Area Municipal Commission of Health and Family Planning (PW2016E-2), Project of Shanghai Municipal Education CommissionGaofeng Clinical Medicine Grant Support (20161412), Key Discipline Project of Shanghai Municipal Commission of Health and Family Planning (15GWZK0701) and Top Priority Clinical Key Disciplines of Shanghai (2017ZZ02016).

\section{Competing Interests}

The authors have declared that no competing interest exists.

\section{References}

[1] Siegel RL, Miller KD, Jemal A. Cancer Statistics, 2017]. CA Cancer J Clin. 2017; 67:7-30.

[2] Wright JD, Barrena Medel NI, Sehouli J, et al. Contemporary management of endometrial cancer]. Lancet. 2012; 379:1352-1360.

[3] Lee S, Vasudevan S. Post-transcriptional stimulation of gene expression by microRNAs [J]. Adv Exp Med Biol. 2013; 768: 97-126.

[4] Srivastava SK, Ahmad A, Zubair $\mathrm{H}$, et al. MicroRNAs in gynecological cancers: Small molecules with big implications]. Cancer letters. 2017; 407:123-138.

[5] Devor EJ, Miecznikowski J, Schickling BM, et al. Dysregulation of miR-181c expression influences recurrence of endometrial endometrioid adenocarcinoma by modulating $\mathrm{NOTCH} 2$ expression: An NRG
Oncology/Gynecologic Oncology Group study. Gynecol Oncol. 2017; 147: 648-653.

[6] Gong B, Yue Y, Wang R, et al. Overexpression of microRNA-194 suppresses the epithelial-mesenchymal transition in targeting stem cell transcription factor Sox3 in endometrial carcinoma stem cells]. Tumour Biol. 2017; 39:1010428317706217.

[7] Zhao X, Zhu D, Lu C, et al. MicroRNA-126 inhibits the migration and invasion of endometrial cancer cells by targeting insulin receptor substrate 1]. Oncol Lett.2016; 11: 1207-1212.

[8] You J, Zhang Y, Li Y, et al. MiR-449a suppresses cell invasion by inhibiting MAP2K1 in non-small cell lung cancer. Am J Cancer Res. 2015; 5:2730-2744.

[9] Li Q, Peng J, Li X, et al. miR-449a targets Flot2 and inhibits gastric cancer invasion by inhibiting TGF-beta-mediated EMT]. Diagn Pathol. 2015; 10: 202.

[10] Chen W, Liu Y, Chen H, et al. Loss of miR-449a-caused PrLZ overexpression promotes prostate cancer metastasis]. Int J Oncol. 2017; 51: 435-444.

[11] Luo W, Huang B, Li Z, et al. MicroRNA-449a is downregulated in non-small cell lung cancer and inhibits migration and invasion by targeting c-Met. PloS one.2013; 8: e64759.

[12] Wei B, Song $Y$, Zhang $Y$, et al. microRNA-449a functions as a tumor-suppressor in gastric adenocarcinoma by targeting Bcl-2 . Oncol Lett. 2013; 6: 1713-1718.

[13] Mangelsdorf DJ, Thummel C, Beato M, et al. The nuclear receptor superfamily: the second decade]. Cell.1995; 83:835-839.

[14] Onate SA, Tsai SY, Tsai MJ, et al. Sequence and characterization of a coactivator for the steroid hormone receptor superfamily. Science. 1995; 270: 1354-1357.

[15] Osborne CK, Bardou V, Hopp TA, et al. Role of the estrogen receptor coactivator AIB1 (SRC-3) and HER-2/neu in tamoxifen resistance in breast cancer. J Natl Cancer Inst. 2003; 95:353-361.

[16] Taylor BS, Schultz N, Hieronymus H, et al. Integrative genomic profiling of human prostate cancer]. Cancer cell. 2010; 18:11-22.

[17] Kershah SM, Desouki MM, Koterba KL, et al. Expression of estrogen receptor coregulators in normal and malignant human endometrium. Gynecol Oncol. 2004; 92:304-313.

[18] Sakaguchi H, Fujimoto J, Sun WS, et al. Clinical implications of steroid receptor coactivator (SRC)-3 in uterine endometrial cancers. J Steroid Biochem Mol Biol. 2007; 104: 237-240.

[19] Balmer NN, Richer JK, Spoelstra NS, et al. Steroid receptor coactivator AIB1 in endometrial carcinoma, hyperplasia and normal endometrium: Correlation with clinicopathologic parameters and biomarkers. Mod Pathol. 2006; 19: 1593-1605.

[20] Rohira AD, Lonard DM. Steroid receptor coactivators present a unique opportunity for drug development in hormone-dependent cancers]. Biochem Pharmacol. 2017; 140: 1-7.

[21] Wang Y, Lonard DM, Yu Y, et al. Small molecule inhibition of the steroid receptor coactivators, SRC-3 and SRC-1. Mol Endocrinol. 2011; 25: 2041-2053.

[22] Song X, Chen J, Zhao M, et al. Development of potent small-molecule inhibitors to drug the undruggable steroid receptor coactivator-3]. Proc Natl Acad Sci U S A. 2016; 113: 4970-4975.

[23] Yin P, Wang Y, Qiu Y, et al. Bufalin-loaded mPEG-PLGA-PLL-cRGD nanoparticles: preparation, cellular uptake, tissue distribution, and anticancer activity]. Int J Nanomedicine. 2012; 7:3961-3969.

[24] Chen J. Is Src the key to understanding metastasis and developing new treatments for colon cancer? Nat Clin Pract Gastroenterol Hepatol. 2008; 5:306-307. 